\title{
Metric Dimension, Minimal Doubly Resolving Sets, and the Strong Metric Dimension for Jellyfish Graph and Cocktail Party Graph
}

\author{
Jia-Bao Liu $\mathbb{D}^{1},{ }^{1}$ Ali Zafari $\mathbb{D}{ }^{2}$ and Hassan Zarei $^{2}$ \\ ${ }^{1}$ School of Mathematics and Physics, Anhui Jianzhu University, Hefei 230601, China \\ ${ }^{2}$ Department of Mathematics, Faculty of Science, Payame Noor University, P.O. Box 19395-4697, Tehran, Iran \\ Correspondence should be addressed to Ali Zafari; zafari.math.pu@gmail.com
}

Received 13 December 2019; Accepted 15 April 2020; Published 29 May 2020

Academic Editor: Jose C. Valverde

Copyright (c) 2020 Jia-Bao Liu et al. This is an open access article distributed under the Creative Commons Attribution License, which permits unrestricted use, distribution, and reproduction in any medium, provided the original work is properly cited.

Let $\Gamma$ be a simple connected undirected graph with vertex set $V(\Gamma)$ and edge set $E(\Gamma)$. The metric dimension of a graph $\Gamma$ is the least number of vertices in a set with the property that the list of distances from any vertex to those in the set uniquely identifies that vertex. For an ordered subset $W=\left\{w_{1}, w_{2}, \ldots, w_{k}\right\}$ of vertices in a graph $\Gamma$ and a vertex $v$ of $\Gamma$, the metric representation of $v$ with respect to $W$ is the $k$-vector $r(v \mid W)=\left(d\left(v, w_{1}\right), d\left(v, w_{2}\right), \ldots, d\left(v, w_{k}\right)\right)$. If every pair of distinct vertices of $\Gamma$ have different metric representations, then the ordered set $W$ is called a resolving set of $\Gamma$. It is known that the problem of computing this invariant is NP-hard. In this paper, we consider the problem of determining the cardinality $\psi(\Gamma)$ of minimal doubly resolving sets of $\Gamma$ and the strong metric dimension for the jellyfish graph JFG $(n, m)$ and the cocktail party graph $\mathrm{CP}(k+1)$.

\section{Introduction}

In this paper, we consider finite, simple, and connected graphs. The vertex and edge sets of a graph $\Gamma$ are denoted by $V(\Gamma)$ and $E(\Gamma)$, respectively. For $u, v \in V(\Gamma)$, the length of a shortest path from $u$ to $v$ is called the distance between $u$ and $v$ and is denoted by $d_{\Gamma}(u, v)$, or simply $d(u, v)$. The adjacency and nonadjacency relations are denoted by $\sim$ and $\nsim$, respectively. The size of the largest clique in the graph $\Gamma$ is denoted by $\omega(\Gamma)$ and the size of the largest independent sets of vertices by $\alpha(\Gamma)$. A vertex $x \in V(\Gamma)$ is said to resolve a pair $u, v \in V(\Gamma)$, if $d_{\Gamma}(u, x) \neq d_{\Gamma}(v, x)$. For an ordered subset $W=\left\{w_{1}, w_{2}, \ldots, w_{k}\right\}$ of vertices in a connected graph $\Gamma$ and a vertex $v$ of $\Gamma$, the metric representation of $v$ with respect to $W$ is the $k$-vector $r(v \mid W)=\left(d\left(v, w_{1}\right), d\left(v, w_{2}\right), \ldots, d(v\right.$, $\left.w_{k}\right)$ ). If every pair of distinct vertices of $\Gamma$ have different metric representations, then the ordered set $W$ is called a resolving set of $\Gamma$. Indeed, the set $W$ is called a resolving set for $\Gamma$ if $r(u \mid W)=r(v \mid W)$ which implies that $u=v$ for all pairs $u, v$ of vertices of $\Gamma$. If the set $W$ is as small as possible, then it is called a metric basis of the graph $\Gamma$. We recall that the metric dimension of $\Gamma$, denoted by $\beta(\Gamma)$, is defined as the minimum cardinality of a resolving set for $\Gamma$. If $\beta(\Gamma)=k$, then $\Gamma$ is said to be $k$-dimensional. Chartrand et al. [1] determined the bounds of the metric dimensions for any connected graphs and determined the metric dimensions of some well-known families of graphs such as trees, paths, and complete graphs. Bounds on $\beta(\Gamma)$ are presented in terms of the order and the diameter of $\Gamma$. All connected graphs of order $n$ having metric dimension $1, n-1$, or $n-2$ are determined. The concept of resolving the set and that of metric dimension date back to the 1950s. They were defined by Bluementhal [2] in the context of metric space. These notions were introduced to graph theory by Harary and Melter [3] and Slater [4] in the 1970s. For more results related to these concepts see [5-10]. These concepts have different applications in the areas of network discovery and verification [11], robot navigation [12], chemistry [1], and combinatorial optimization [13]. Notice for each connected graph $\Gamma$ and each ordered set $W=\left\{w_{1}, w_{2}, \ldots, w_{k}\right\}$ of vertices of $\Gamma$ that the $i^{\text {th }}$ coordinate of $r\left(w_{i} \mid W\right)$ is 0 and that the $i^{\text {th }}$ coordinate of all other vertex representations is positive. Thus, certainly $r(u \mid W)=r(v \mid W)$ implies that $u=v$ for $u \in W$. Therefore, when testing whether an ordered subset $W$ of $V(\Gamma)$ is a resolving set for $\Gamma$, we need only be concerned with the vertices of $V(\Gamma)-W$. 
Cáceres et al. [14] define the notion of a doubly resolving set as follows. Vertices $x, y$ of the graph $\Gamma$ of order at least 2 are said to doubly resolve vertices $u, v$ of $\Gamma$ if $d(u, x)-d(u, y) \neq d(v, x)-d(v, y) . \quad$ A $\quad$ set $Z=\left\{z_{1}, z_{2}, \ldots, z_{l}\right\}$ of vertices of $\Gamma$ is a doubly resolving set of $\Gamma$ if every two distinct vertices of $\Gamma$ are doubly resolved by some two vertices of $Z$. The minimal doubly resolving set is a doubly resolving set with minimum cardinality. The cardinality of the minimum doubly resolving set is denoted by $\psi(\Gamma)$. The minimal doubly resolving sets for Hamming and prism graphs have been obtained in $[15,16]$, respectively. Other researchers in [17] determined the minimal doubly resolving sets for the necklace graph. Since if $x, y$ doubly resolve $u, v$, then $d(u, x)-d(v, x) \neq 0 \quad$ or $d(u, y)-d(v, y) \neq 0$, and hence $x$ or $y$ resolve $u, v$. Therefore, a doubly resolving set is also a resolving set, and $\beta(\Gamma) \leq \psi(\Gamma)$.

The strong metric dimension problem was introduced by Sebö and Tannier [13] and further investigated by Oellermann and Peters-Fransen [18]. Recently, the strong metric dimension of distance hereditary graphs has been studied by May and Oellermann [19]. For more results related to this concept see [20]. A vertex $w$ strongly resolves two vertices $u$ and $v$ if $u$ belongs to a shortest $v-w$ path or $v$ belongs to a shortest $u-w$ path. A set $N=\left\{n_{1}, n_{2}, \ldots, n_{m}\right\}$ of vertices of $\Gamma$ is a strong resolving set of $\Gamma$ if every two distinct vertices of $\Gamma$ are strongly resolved by some vertex of $N$. A strong metric basis of $\Gamma$ is a strong resolving set of the minimum cardinality. Now, the strong metric dimension of $\Gamma$, denoted by $\operatorname{sdim}(\Gamma)$, is defined as the cardinality of its strong metric basis. It is easy to see that if a vertex $w$ strongly resolves vertices $u$ and $v$, then $w$ also resolves these vertices. Hence, every strong resolving set is a resolving set, and $\beta(\Gamma) \leq \operatorname{sdim}(\Gamma)$.

All three previously defined problems are NP-hard in general case. The proofs of NP-hardness are given for the metric dimension problem in [12], for the minimal doubly resolving set problem in [21], and for the strong metric dimension problem in [18]. Intrinsic metrics on a graph have become of interest, as generally discussed in [5,16,22-25], for some classes of graphs. In this paper, we consider the problem of determining the cardinality $\psi(\Gamma)$ of minimal doubly resolving sets of $\Gamma$ and the strong metric dimension for the jellyfish graph JFG $(n, m)$ and the cocktail party graph $\mathrm{CP}(k+1)$. In fact, we find the minimum cardinality of the resolving set, doubly resolving set, and strong resolving set of the jellyfish graph JFG $(n, m)$. In particular, we show that the minimum cardinality of the resolving set, doubly resolving set, and strong resolving set of the cocktail party graph $\mathrm{CP}(k+1)$ is $k+1$.

\section{Definitions and Preliminaries}

Definition 1 (see [26]). Let $\Gamma$ be a graph with automorphism group Aut $(\Gamma)$. We say that $\Gamma$ is a vertex transitive graph if, for any vertices $x, y$ of $\Gamma$, there is some $\varphi$ in $\operatorname{Aut}(\Gamma)$, such that $\varphi(x)=y$. Also, we say that $\Gamma$ is symmetric if, for all vertices $u, v, x, y$ of $\Gamma$ such that $u$ and $v$ are adjacent, and also, $x$ and $y$ are adjacent, there is an automorphism $\varphi$ such that $\varphi(u)=x$ and $\varphi(v)=y$. Finally, we say that $\Gamma$ is a distance transitive if, for all vertices $u, v, x, y$ of $\Gamma$ such that $d(u, v)=d(x, y)$, there is an automorphism $\varphi$ such that $\varphi(u)=x$ and $\varphi(v)=y$.

Definition 2 (see [26]). Let $G$ be a finite group and $\Omega$ a subset of $G$ that is closed under taking inverses and does not contain the identity. A Cayley graph $\Gamma=\operatorname{Cay}(G, \Omega)$ is a graph whose vertex set and edge set are defined as follows:

$$
\begin{aligned}
& V(\Gamma)=G ; \\
& E(\Gamma)=\left\{\{x, y\} \mid x^{-1} y \in \Omega\right\} .
\end{aligned}
$$

Remark 1 (see [26]). Let $\Gamma$ be a graph. It is clear that we have a hierarchy of the conditions as follows:

distance transitive $\Rightarrow$ symmetric $\Rightarrow$ vertex transitive.

Proposition 1 (see [27]). Let $\Gamma=\operatorname{Cay}\left(\mathbb{Z}_{n}, S_{k}\right)$ be the Cayley graph on the cyclic group $\mathbb{Z}_{n}(n \geq 4)$, where $S_{1}=\{1, n-1\}, \ldots, S_{k}=S_{k-1} \cup\{k, n-k\}$ are the inverse closed subsets of $\mathbb{Z}_{n}-\{0\}$ for any $k \in \mathbb{N}, 1 \leq k \leq[n / 2]-1$. Then, $\chi(\Gamma)=\omega(\Gamma)=k+1$ if and only if $k+1 \mid n$, where the chromatic number $\chi(\Gamma)$ of $\Gamma$ is the minimum number $k$ such that $\Gamma$ is $k$ colorable.

Definition 3 (see [23]). Let $\Gamma$ be a graph, and let $W=\left\{w_{1}, \ldots, w_{k}\right\} \subseteq V(\Gamma)$. For each vertex $v \in V(\Gamma)$, the adjacency representation of $v$ with respect to $W$ is the $k$-vector:

$$
\widehat{r}(v \mid W)=\left(a_{\Gamma}\left(v, w_{1}\right), \ldots, a_{\Gamma}\left(v, w_{k}\right)\right),
$$

where

$$
a_{\Gamma}\left(v, w_{i}\right)= \begin{cases}0, & \text { if } v=w_{i} \\ 1, & \text { if } v \sim w_{i} \\ 2, & \text { if } v+w_{i}\end{cases}
$$

The set $W$ is an adjacency resolving set for $\Gamma$ if the vectors $\widehat{r}(v \mid W)$ for $v \in V(\Gamma)$ are distinct. The minimum cardinality of an adjacency resolving set is the adjacency dimension of $\Gamma$, denoted by $\widehat{\beta}(\Gamma)$. An adjacency resolving set of cardinality $\widehat{\beta}(\Gamma)$ is an adjacency basis of $\Gamma$.

Lemma 1 (see [23]). Let $\Gamma$ be a graph of order $n$.

(1) If $\operatorname{diam}(\Gamma)=2$, then $\widehat{\beta}(\Gamma)=\beta(\Gamma)$.

(2) If $\Gamma$ is connected, then $\widehat{\beta}(\Gamma)=\beta(\Gamma)$.

(3) $1 \leq \widehat{\beta}(\Gamma) \leq n-1$.

\section{Main Results}

3.1. Metric Dimension, Minimal Doubly Resolving Sets, and the Strong Metric Dimension of the Jellyfish Graph JFG $(n, m)$. For an integer $n \geq 3$, the $n$-cycle is the graph $C_{n}$ with vertex set $V\left(C_{n}\right)=\left\{v_{1}, v_{2}, \ldots, v_{n}\right\}$ or simply $\{1,2, \ldots, n\}$ and edge set $E\left(C_{n}\right)=\left\{v_{i} v_{i+1}: 1 \leq i \leq n\right\}$, where $v_{n+1}=v_{1}$. An interesting family of graphs of order $n m+n$ is defined as follows. Let $\Gamma$ be a graph with a vertex set $V_{1} \cup V_{2}$, where $V_{1}=V\left(C_{n}\right)$ and $V_{2}=\left\{A_{1 j}, A_{2 j}, \ldots, A_{n j}\right\}$, and let $A_{i j}=\cup_{j=1}^{m} v_{i j}, \quad 1 \leq i \leq n$. 
Suppose that every vertex $i \in V_{1}$ is adjacent to vertices $v_{i 1}, v_{i 2}, \ldots, v_{i m} \in A_{i j}$ and $\operatorname{deg}\left(v_{i j}\right)=1$ for every vertex $v_{i j} \in A_{i j}$, then the resulting graph is called the jellyfish graph JFG $(n, m)$ with parameters $m$ and $n$. In particular, if $n$ is an even integer, then the jellyfish graph $\operatorname{JFG}(n, m)$ is a bipartite graph. In this paper, we consider the problem of determining the cardinality $\psi(\operatorname{JFG}(n, m))$ of minimal doubly resolving sets of the jellyfish graph JFG $(n, m)$. First, we find the metric dimension of the jellyfish graph JFG $(n, m)$, in fact we prove that if $n \geq 3$ and $m \geq 2$, then the metric dimension of the jellyfish graph JFG $(n, m)$ is $n m-n$. Also, we consider the problem of determining the cardinality $\psi(\operatorname{JFG}(n, m))$ of minimal doubly resolving sets of JFG $(n, m)$ and the strong metric dimension of the jellyfish graph JFG $(n, m)$. Moreover, we find an adjacency dimension of the jellyfish graph JFG $(n, m)$.

Theorem 1. Let $n, m$ be integers such that $n \geq 3$ and $m \geq 2$. Then, the metric dimension of the jellyfish graph $\operatorname{JFG}(n, m)$ is $n m-n$.

Proof. Let $V(\operatorname{JFG}(n, m))=V_{1} \cup V_{2}$, where $V_{1}=V\left(C_{n}\right)=$ $\{1,2, \ldots, n\}$ and $V_{2}=\left\{A_{1 j}, A_{2 j}, \ldots, A_{n j}\right\}$, and let $A_{i j}=\cup_{j=1}^{m} v_{i j}, 1 \leq i \leq n$. Suppose that every vertex $i \in V_{1}$ is adjacent to vertices $v_{i 1}, v_{i 2}, \ldots, v_{i m} \in A_{i j}$. We can show that the diameter of the jellyfish graph JFG $(n, m)$ is $[n / 2]+2$. In the following cases, we show that the metric dimension of the jellyfish graph JFG $(n, m)$ is $n m-n$.

Case 1. Let $W$ be an ordered subset of $V_{1}$ in the jellyfish graph JFG $(n, m)$ such that $|W| \leq n$. It is easy to prove that if $|W| \leq n$, then $W$ is not a resolving set of the jellyfish graph JFG $(n, m)$. In particular, if $|W|=n$, then we show that $W$ is not a resolving set of the jellyfish graph JFG $(n, m)$. We may assume without loss of generality that an ordered subset is $W=\{1,2, \ldots, n\}$. Hence, $\quad V(\operatorname{JFG}(n, m))-W=\left\{A_{1 j}, A_{2 j}, \ldots, A_{n j}\right\}$. Therefore, the metric representation of the vertices $v_{11}, v_{12}, \ldots, v_{1 m} \in A_{1 j}$ with respect to $W$ is the same as the $n$-vector. Thus, $W$ is not a resolving set of the jellyfish graph JFG $(n, m)$.

Case 2. Let $W$ be an ordered subset of $V_{2}$ in the jellyfish graph JFG $(n, m)$ such that $W=\left\{A_{2 j}, A_{3 j}, \ldots, A_{n j}\right\}$. Hence, $V(\operatorname{JFG}(n, m))-W=\left\{1,2, \ldots, n, A_{1 j}\right\}$. We know that $|W|=n m-m$. Therefore, the metric representation of the vertices $v_{11}, v_{12}, \ldots, v_{1 m} \in A_{1 j}$ with respect to $W$ is the same as the $n m-m$-vector. Thus, $W$ is not a resolving set of jellyfish graph JFG $(n, m)$.

Case 3. Let $W$ be an ordered subset of $V_{2}$ in the jellyfish graph JFG $(n, m)$ such that $W=\left\{A_{1 j}, A_{2 j}\right.$, $\left.A_{3 j}, \ldots, A_{n j}-\left\{v_{n 1}, v_{n 2}\right\}\right\}$. Hence, $V(\operatorname{JFG}(n, m))-W$ $=\left\{1,2, \ldots, n, v_{n 1}, v_{n 2}\right\}$. We know that $|W|=n m-2$. Therefore, the metric representation of the vertices $v_{n 1}, v_{n 2} \in A_{n j}$ with respect to $W$ is the same as the $n m-2$-vector. Thus, $W$ is not a resolving set of the jellyfish graph JFG $(n, m)$.

Case 4. Let $W$ be an ordered subset of $V_{2}$ in the jellyfish graph JFG $(n, m)$ such that $|W|=n m-1$. We show that
$W$ is a resolving set of the jellyfish graph JFG $(n, m)$. We may assume without loss of generality that an ordered subset is $W=\left\{A_{1 j}, A_{2 j}, \ldots, A_{n j}-v_{n m}\right\}$. Hence, $V(\operatorname{JFG}(n, m))-W=\left\{1,2, \ldots, n, v_{n m}\right\}$. We can show that all the vertices $1,2, \ldots, n, v_{n m} \in V(\operatorname{JFG}(n, m))-$ $W$ have different representations with respect to $W$. For every $k \in V(\operatorname{JFG}(n, m))-W, 1 \leq k \leq n$ and $v_{i j} \in A_{i j}$, $1 \leq i \leq n$, and $1 \leq j \leq m$, if $k=i$, then we have $d\left(k, v_{i j}\right)=1$, otherwise $d\left(k, v_{i j}\right)>1$. Also, for the vertex $v_{n m} \in V(\operatorname{JFG}(n, m))-W$ with $v_{n m} \neq v_{i j} \in A_{i j}, 1 \leq i \leq n$, and $1 \leq j \leq m$, if $i=n$, then we have $d\left(v_{n m}, v_{i j}\right)=2$, otherwise $d\left(v_{n m}, v_{i j}\right)>2$. Therefore, all the vertices $1,2, \ldots, n, v_{n m} \in V(\operatorname{JFG}(n, m))-W$ have different representations with respect to $W$. This implies that $W$ is a resolving set of the jellyfish graph JFG $(n, m)$.

Case 5. Let $W$ be an ordered subset of $V_{2}$ in the jellyfish graph JFG $(n, m)$ such that $W=\left\{A_{1 j}-v_{1 m}\right.$, $\left.A_{2 j}-v_{2 m}, \ldots, A_{n j}-v_{n m}\right\}$. Hence, $V(\operatorname{JFG}(n, m))-W$ $=\left\{1,2, \ldots, n, v_{1 m}, v_{2 m}, \ldots, v_{n m}\right\}$. We know that $|W|=n m-n$. In a similar fashion which is done in Case 4 , we can show that all the vertices $1,2, \ldots$ $, n, v_{1 m}, v_{2 m}, \ldots, v_{n m} \in V(\operatorname{JFG}(n, m))-W$ have different representations with respect to $W$. This implies that $W$ is a resolving set of the jellyfish graph $\operatorname{JFG}(n, m)$.

Case 6. In particular, let $W$ be an ordered subset of $V_{2}$ in the jellyfish graph JFG $(n, m)$ such that $|W|=n m$. We show that $W$ is a resolving set of the jellyfish graph $\operatorname{JFG}(n, m)$. We may assume without loss of generality that an ordered subset is $W=\left\{A_{1 j}, A_{2 j}, \ldots, A_{n j}\right\}$. Hence, $V(\operatorname{JFG}(n, m))-W=\{1,2, \ldots, n\}$. We can show that all the vertices $1,2, \ldots, n \in V(\operatorname{JFG}(n, m))-$ $W$ have different representations with respect to $W$. This implies that $W$ is a resolving set of the jellyfish graph JFG $(n, m)$.

From the above cases, we conclude that the minimum cardinality of a resolving set of the jellyfish graph JFG $(n, m)$ is $n m-n$.

Lemma 2. Let $n$ and $m$ be integers such that $n \geq 3$ and $m \geq 2$. Then, the subset $Z=\left\{A_{1 j}-v_{1 m}, A_{2 j}-v_{2 m}, \ldots, A_{n j}-v_{n m}\right\}$ of vertices in the jellyfish graph JFG $(n, m)$ is not a doubly resolving set of the jellyfish graph JFG $(n, m)$.

Proof. We know that the ordered subset $Z=\left\{A_{1 j}-v_{1 m}, A_{2 j}-v_{2 m}, \ldots, A_{n j}-v_{n m}\right\}$ of vertices in the jellyfish graph JFG $(n, m)$ is a resolving set of the jellyfish graph JFG $(n, m)$ of size $n m-n$. Also by Theorem 1, the metric dimension of the jellyfish graph $\operatorname{JFG}(n, m)$ is $\beta(\operatorname{JFG}(n, m))=n m-n . \quad$ Moreover, $\quad B(\operatorname{JFG}(n, m)) \leq \psi$ $(\operatorname{JFG}(n, m))$. We show that the subset $Z=\left\{A_{1 j}-v_{1 m}, A_{2 j}-\right.$ $\left.v_{2 m}, \ldots, A_{n j}-v_{n m}\right\}$ of vertices in the jellyfish graph JFG $(n, m)$ is not a doubly resolving set of the jellyfish graph JFG $(n, m)$. If $u=v_{i m}$ and $v=i$ and $1 \leq i \leq n$, then for every $x, y \in Z$, we have $d(u, x)-d(u, y)=d(v, x)-d(v, y)$.

Lemma 3. Let $n$ and $m$ be integers such that $n \geq 3$ and $m \geq 2$. Then, the subset $Z=\left\{A_{1 j}, A_{2 j}, \ldots, A_{n j}-v_{n m}\right\}$ of vertices in 
the jellyfish graph JFG $(n, m)$ is not a doubly resolving set of the jellyfish graph JFG $(n, m)$.

Proof. We show that the subset $Z=\left\{A_{1 j}, A_{2 j}, \ldots, A_{n j}-v_{n m}\right\}$ of vertices in the jellyfish graph JFG $(n, m)$ is not a doubly resolving set of the jellyfish graph $\operatorname{JFG}(n, m)$. If $u=v_{n m}$ and $v=n$, then for every $x, y \in Z$, we have $d(u, x)-d(u, y)=d(v, x)-d(v, y)$.

Theorem 2. Let $n$ and $m$ be integers such that $n \geq 3$ and $m \geq 2$. Then, the cardinality of the minimum doubly resolving set of the jellyfish graph JFG $(n, m)$ is $n m$.

Proof. Let $V(\operatorname{JFG}(n, m))=V_{1} \cup V_{2}$, where $V_{1}=V\left(C_{n}\right)=$ $\{1,2, \ldots, n\}$ and $V_{2}=\left\{A_{1 j}, A_{2 j}, \ldots, A_{n j}\right\}$, and let $A_{i j}=\cup_{j=1}^{m} v_{i j}, 1 \leq i \leq n$. Suppose that every vertex $i \in V_{1}$ is adjacent to vertices $v_{i 1}, v_{i 2}, \ldots, v_{i m} \in A_{i j}$. We know that the ordered subset $v_{i 1}, v_{i 2}, \ldots, v_{i m} \in A_{i j}$ of vertices in the jellyfish graph JFG $(n, m)$ is a resolving set of the jellyfish graph $\operatorname{JFG}(n, m)$ of size $n m$. Also by Theorem 1 , the metric dimension of the jellyfish graph $\operatorname{JFG}(n, m)$ is $\operatorname{JFG}(n, m)$. Moreover, $B(\operatorname{JFG}(n, m)) \leq \psi(\operatorname{JFG}(n, m))$. We show that the subset $Z=\left\{A_{1 j}, A_{2 j}, \ldots, A_{n j}\right\}$ of vertices in the jellyfish graph JFG $(n, m)$ is a doubly resolving set of the jellyfish graph JFG $(n, m)$. It is sufficient to show that for two vertices $u$ and $v$ of the jellyfish graph JFG $(n, m)$ there are vertices $x, y \in Z$ such that $d(u, x)-d(u, y) \neq d(v, x)-d(v, y)$. Consider two vertices $u$ and $v$ of the jellyfish graph JFG $(n, m)$. Then, we have the following:

Case 1. Let $u \notin Z$ and $v \notin Z$. Hence, $u, v \in V_{1}=V\left(C_{n}\right)=\{1,2, \ldots, n\}$. We can assume without loss of generality that $u=i$ and $v=j$, $1 \leq i, j \leq n$, and $i \neq j$. Therefore, if $x=v_{i 1}$ and $y=v_{j 1}$, and then we have $d(u, x)-d(u, y) \neq d(v, x)-d(v, y)$ because $d(u, x)-d(u, y)<0$ and $d(v, x)-d(v, y)>0$.

Case 2. Let $u \in Z$ and $v \in Z$. Hence, $u, v \in V_{2}=\left\{A_{1 j}, A_{2 j}, \ldots, A_{n j}\right\}$. Therefore, if $x=u$ and $y=v$, then we have $d(u, x)-d(u, y)$ $\neq d(v, x)-d(v, y)$ because $d(u, x)-d(u, y)<0$ and $d(v, x)-d(v, y)>0$.

Case 3. Finally, let $u \notin Z$ and $v \in Z$. Hence, $u \in V_{1}=$ $V\left(C_{n}\right)=\{1,2, \ldots, n\}$ and $v \in V_{2}=\left\{A_{1 j}, A_{2 j}, \ldots, A_{n j}\right\}$. We can assume without loss of generality that $u=k$, $1 \leq k \leq n$, and $v=v_{11} \in A_{11}$. Therefore, if $x=v_{k 2}$ and $y=v_{11}$, then we have $d(u, x)-d(u, y) \neq d(v, x)$ $-d(v, y)$ because $d(u, x)-d(u, y) \leq 0 \quad$ and $d(v, x)-d(v, y)>0$.

Thus, by Lemmas 2 and 3 and the above cases, we conclude that the cardinality of the minimum doubly resolving set of the jellyfish graph JFG $(n, m)$ is $n m$.

Lemma 4. Let $n$ and $m$ be integers such that $n \geq 3$ and $m \geq 2$. Then, the subset $N=\left\{A_{1 j}-v_{1 m}, A_{2 j}-v_{2 m}, \ldots, A_{n j}-v_{n m}\right\}$ of vertices in the jellyfish graph JFG $(n, m)$ is not a strong resolving set of the jellyfish graph JFG $(n, m)$.
Proof. Let $M=V_{2}-N=\left\{v_{1 m}, v_{2 m}, \ldots, v_{n m}\right\}$, where $V_{2}$ is the set which is defined already. It is not hard to see that for every two distinct vertices $u, v \in M$, there is not a vertex $w \in N$ such that $u$ belongs to a shortest $v-w$ path or $v$ belongs to a shortest $u-w$ path. So, the subset $N=\left\{A_{1 j}-v_{1 m}, A_{2 j}-v_{2 m}, \ldots, A_{n j}-v_{n m}\right\}$ of vertices in the jellyfish graph $\operatorname{JFG}(n, m)$ is not a strong resolving set of the jellyfish graph JFG $(n, m)$. We conclude that if $N$ is a strong resolving set of the jellyfish graph $\operatorname{JFG}(n, m)$, then $|N| \geq n m-1$ because $|M|$ must be less than 2 .

Theorem 3. Let $n$ and $m$ be integers such that $n \geq 3$ and $m \geq 2$. Then, the strong metric dimension of the jellyfish graph $J F G(n, m)$ is $n m-1$.

Proof. By Lemma 4, we know that if $N$ is a strong resolving set of the jellyfish graph $\operatorname{JFG}(n, m)$, then $|N| \geq n m-1$. We show that the subset $N=\left\{A_{1 j}, A_{2 j}, \ldots, A_{n j}-v_{n m}\right\}$ of vertices in the jellyfish graph JFG $(n, m)$ is a strong resolving set of the jellyfish graph JFG $(n, m)$. It is sufficient to prove that every two distinct vertices $u, v \in V$ (JFG $(n$, $m))-N=\left\{1,2, \ldots, n, v_{n m}\right\}$ are strongly resolved by a vertex $w \in N$. In the following cases, we show that the strong metric dimension of the jellyfish graph JFG $(n, m)$ is $n m-1$.

Case 1. Let $u$ and $v$ be two distinct vertices in $V(\operatorname{JFG}(n, m))-N$ such that $u, v \in V_{1}=V\left(C_{n}\right)$ $=\{1,2, \ldots, n\}$. So, there is $i, j \in V_{1}$ such that $u=i$ and $v=j$. Therefore, $i$ and $j$ will be strongly resolved by some $v_{i j} \in A_{i j}$ because $i$ and $v_{i j}$ are adjacent, and hence $i$ belongs to a shortest $v_{i j}-j$ path.

Case 2. Now, let $u$ and $v$ be two distinct vertices in $V(\operatorname{JFG}(n, m))-N$ such that $u \in V_{1}=\{1,2, \ldots, n\}$ and $v=v_{n m}$. Without loss of generality we may assume $u=i$, where $i \in V_{1}$. Therefore, $i$ and $v_{n m}$ will be strongly resolved by some $v_{i j} \in A_{i j}$ because $i$ and $v_{i j}$ are adjacent, and hence $i$ belongs to a shortest $v_{i j}-v_{n m}$ path.

From the above cases, we conclude that the minimum cardinality of a strong metric dimension of the jellyfish graph JFG $(n, m)$ is $n m-1$.

Lemma 5. Let $n$ and $m$ be integers such that $n \geq 3$ and $m \geq 2$. Then, an ordered subset $W=\left\{A_{1 j}-v_{1 m}, A_{2 j}-v_{2 m}, \ldots, A_{n j}-\right.$ $\left.v_{n m}\right\}$ of vertices in the jellyfish graph JFG $(n, m)$ is not the adjacency resolving set of the jellyfish graph JFG $(n, m)$.

Proof. Let $\Gamma=\operatorname{JFG}(n, m)$ and $\quad M=V_{2}-W=\left\{v_{1 m}\right.$, $\left.v_{2 m}, \ldots, v_{n m}\right\}$, where $V_{2}$ is the set which is defined already. Thus, the adjacency representation of the vertices $v_{1 m}, v_{2 m}, \ldots, v_{n m} \in V(\operatorname{JFG}(n, m))-W$ with respect to $W$ is the $n m-n$-vector $\quad \widehat{r}\left(v_{1 m} \mid W\right)=\widehat{r}\left(v_{2 m} \mid W\right)=\ldots=\hat{r}$ $\left(v_{n m} \mid W\right)=(2,2, \ldots, 2)$. For every vertex $w \in W$, we have $a_{\Gamma}\left(w, v_{1 m}\right)=a_{\Gamma}\left(w, v_{1 m}\right)=\ldots=a_{\Gamma}\left(w, v_{n m}\right)=2$. We conclude that if $W$ is an adjacency resolving set of the jellyfish graph JFG $(n, m)$, then $|W| \geq n m-1$ because $|M|$ must be less than 2. 
Theorem 4. Let $n$ and $m$ be integers such that $n \geq 3$ and $m \geq 2$. Then, the adjacency dimension of the jellyfish graph $J F G(n, m)$ is $n m-1$.

Proof. By Lemma 5, we know that if $W$ is an adjacency resolving set of the jellyfish graph $\operatorname{JFG}(n, m)$, then $|W| \geq n m-1$. Now, let $\Gamma=\operatorname{JFG}(n, m)$ and $W$ be an ordered subset of $V_{2}$ in the jellyfish graph $\operatorname{JFG}(n, m)$ such that $|W|=n m-1$. We show that $W$ is an adjacency resolving set of the jellyfish graph JFG $(n, m)$. We may assume without loss of generality that an ordered subset is $W=\left\{A_{1 j}, A_{2 j}, \ldots, A_{n j}-v_{n m}\right\}$. Hence, $V(\operatorname{JFG}(n, m))-W=$ $\left\{1,2, \ldots, n, v_{n m}\right\}$. We can show that all the vertices $1,2, \ldots, n, v_{n m} \in V(\operatorname{JFG}(n, m))-W$ have different adjacency representations with respect to $W$. For every $k \in V(\operatorname{JFG}(n, m))-W, 1 \leq k \leq n$ and $v_{i j} \in A_{i j}, 1 \leq i \leq n$, and $1 \leq j \leq m$, if $k=i$, then we have $a_{\Gamma}\left(k, v_{i j}\right)=1$, otherwise $a_{\Gamma}\left(k, v_{i j}\right)=2$. Also, for the vertex $v_{n m} \in V(\operatorname{JFG}(n, m))-W$ with $v_{n m} \neq v_{i j} \in A_{i j}, \quad 1 \leq i \leq n$, and $1 \leq j \leq m$, we have $a_{\Gamma}\left(v_{n m}, v_{i j}\right)=2$. Therefore, all the vertices $1,2, \ldots, n, v_{n m} \in V(\operatorname{JFG}(n, m))-W$ have different adjacency representations with respect to $W$. This implies that $W$ is an adjacency resolving set of the jellyfish graph JFG $(n, m)$. We now conclude that the minimum cardinality of the adjacency resolving set of the jellyfish graph $\operatorname{JFG}(n, m)$ is $n m-1$.

3.2. Metric Dimension, Minimal Doubly Resolving Sets, and the Strong Metric Dimension of the Cocktail Party Graph $C P(k+1)$. Let $\Gamma=\operatorname{Cay}\left(\mathbb{Z}_{n}, S_{k}\right)$ be the Cayley graph on the cyclic additive group $\mathbb{Z}_{n}$, where $S_{1}=\{1, n-1\}$, $\ldots, S_{k}=S_{k-1} \cup\{k, n-k\}$ are the inverse closed subsets of $\mathbb{Z}_{n}-\{0\}$ for any $k \in \mathbb{N}, 1 \leq k \leq[n / 2]-1$. If $n$ is an even integer and $k=(n / 2)-1$, then we can show that Cay $\left(\mathbb{Z}_{n}, S_{k}\right)$ is obtained from the complete graph $K_{2(k+1)}$ by deleting a perfect matching, and hence Cay $\left(\mathbb{Z}_{n}, S_{k}\right)$ is isomorphic to the cocktail party graph $\mathrm{CP}(k+1)$. Moreover, we can show that $\operatorname{Cay}\left(\mathbb{D}_{2 n}, \Omega\right)$, where

$$
\mathbb{D}_{2 n}=\left\langle a, b \mid a^{n}=b^{2}=1, b a=a^{n-1} b\right\rangle,
$$

is the dihedral group of order $2 n$ and $\Omega=\left\{a, a^{2}, \ldots, a^{n-1}, a b, a^{2} b, \ldots, a^{n-1} b\right\}$ is the inverse closed subset of $\mathbb{D}_{2 n}-\{1\}$, is isomorphic to the cocktail party graph $\mathrm{CP}(n)$. In this section, we consider the problem of determining the cardinality $\psi(\Gamma)$ of minimal doubly resolving sets of $\Gamma$. First, we show that if $n$ is an even integer and $k=(n / 2)-1$, then the metric dimension of $\Gamma$ is $k+1$. Also, we prove that if $n$ is an even integer and $k=(n / 2)-1$, then every minimal resolving set of $\Gamma$ is also a doubly resolving set, and, consequently, $\psi(\Gamma)$ is equal to the metric dimension of $\beta(\Gamma)$, which is known from the literature. Moreover, we find an explicit expression for the strong metric dimension of $\Gamma$.

Theorem 5. Let $\Gamma=\operatorname{Cay}\left(\mathbb{Z}_{n}, S_{k}\right)$ be the Cayley graph on the cyclic group $\mathbb{Z}_{n}(n \geq 8)$, where $S_{1}=\{1, n-1\}, \ldots, S_{k}=S_{k-1}$ $\cup\{k, n-k\}$ are the inverse closed subsets of $\mathbb{Z}_{n}-\{0\}$ for any $k \in \mathbb{N}, 1 \leq k \leq[n / 2]-1$. If $n$ is an even integer and $k=(n / 2)-1$, then the metric dimension of $\Gamma$ is $k+1$.
Proof. Let $V(\Gamma)=\{1, \ldots, n\}$ be the vertex set of $\Gamma$. By proof of Proposition 3.4 in [27], we know that the diameter of $\Gamma$ is 2. Hence, for all the vertices $x, y \in V(\Gamma)$, the length of a shortest path from $x$ to $y$ is $d(x, y)=1$ or 2 . Also, we know that the size of the largest clique in the graph $\Gamma$ is $k+1$. Now, let $W$ be an ordered subset of vertices in the graph $\Gamma$ such that $W$ is a clique in $\Gamma$. Indeed, for every $x, y \in W$, we have $d_{\Gamma}(x, y)=1$. In the following cases, we show that the metric dimension of $\Gamma$ is $k+1$.

Case 1. It is easy to see that if $|W| \leq k$, then $W$ is not a resolving set of $\Gamma$. In particular, if $|W|=k$, then we show that $W$ is not a resolving set of $\Gamma$. We may assume without loss of generality that an ordered subset of vertices in the graph $\Gamma$ is $W=\{1,2,3, \ldots, k\}$. Hence, $V(\Gamma)-W=\{k+1, k+2, \ldots, n\}$. On the contrary, by proof of Proposition 3.2 in [27], we know that for every vertex $x$ in $\Gamma$, there is exactly one $y$ in $\Gamma$ such that $x^{-1} y=k+1$, that is, $d(x, y)=2$. Hence, there are vertices $k+2, k+3, \ldots, n-1$ in $V(\Gamma)-W$ such that $d(1, k+2)=2, d(2, k+3)=2, \ldots, d(k, n-1)=2$.

Therefore, the metric representations of the vertices $k+$ $2, k+3, \ldots, n-1 \in V(\Gamma)-W$ with respect to $W$ are the $\quad k$-vectors $\quad r(k+2 \mid W)=(2,1,1, \ldots, 1), r$ $(k+3 \mid W)=(1,2,1, \ldots, 1), \ldots, r(n \mid W)=(1,1,1, \ldots$ ,2). Moreover, the metric representation of the vertices $k+1, n \in V(\Gamma)-W$ with respect to $W$ is the $k$-vector $r(k+1 \mid W)=r(n \mid W)=(1,1,1, \ldots, 1)$. Thus, $W$ is not a resolving set of $\Gamma$ because the metric representation of the vertices $k+1, n$ is the same as the $k$-vector.

Case 2. Let $W$ be a clique in the graph $\Gamma$ such that $x \in W$ and $|W|=k$. We know that there is exactly one $y$ in $V(\Gamma)-W$ such that $x^{-1} y=k+1$, that is, $d(x, y)=2$. In the following, we show that an ordered subset $(W \cup y)$ of vertices in the graph $\Gamma$ is not a resolving set of $\Gamma$. In this case, we may assume without loss of generality that an ordered subset of vertices in the graph $\Gamma$ is $W=\{1,2, \ldots, k\}$, and let $x=1$ and $y=k+2$. Therefore, the metric representation of the vertices $k+1, n \in V(\Gamma)-(W \cup y)$ with respect to $(W \cup y)$ is the $k+1$-vector $r(k+1 \mid W)=r(n \mid W)=(1,1,1, \ldots, 1)$. Thus, $(W \cup y)$ is not a resolving set of $\Gamma$.

Case 3. Now, let $W$ be a clique in the graph $\Gamma$ such that $|W|=k+1$. We show that $W$ is a resolving set of $\Gamma$. We may assume without loss of generality that an ordered subset of vertices in the graph $\Gamma$ is $W=\{1,2,3, \ldots, k, k+1\}$. Hence, $V(\Gamma)-W=\{k+2$, $k+3, \ldots, n\}$. Therefore, the metric representations of the vertices $k+2, k+3, \ldots, n \in V(\Gamma)-W$ with respect to $W$ are the $(k+1)$-vectors $r(k+2 \mid W)=(2,1,1$, $\ldots, 1), r(k+3 \mid W) \quad=(1,2,1, \ldots, 1), \ldots, r(n \mid W)=$ $(1,1,1, \ldots, 2)$. Thus, all the vertices of $V(\Gamma)-W$ have different representations with respect to $W$. This implies that $W$ is a resolving set of $\Gamma$. Moreover, the metric dimension of $\beta(\Gamma) \leq k+1$ because $\Gamma$ is a vertex transitive graph.

Case 4. In particular, let $W$ be a clique in the graph $\Gamma$ such that $|W|=k+1$. We show that for each 
$x \in V(\Gamma)-W$, an ordered subset $(W \cup x)$ of vertices in the graph $\Gamma$ is also a resolving set of $\Gamma$. We may assume without loss of generality that an ordered subset of vertices in the graph $\Gamma$ is $W=\{1,2,3, \ldots, k, k+1\}$, and let $x=k+2$. So, $(W \cup x)=\{1,2,3, \ldots, k+1, k+2\}$. Hence, $V(\Gamma)-(W \cup x)=\{k+3, k+4, \ldots, n\}$. Therefore, the metric representations of the vertices $k+3, k+4, \ldots, n \in V(\Gamma)-(W \cup x)$, with respect to $(W \cup x)$, are the $k+2$-vectors $r(k+3 \mid W)=(1,2,1$, $\ldots, 1,1), r(k+4 \mid W)=(1,2,1, \ldots, 1,1), \ldots, r(n \mid W)$ $=(1,2,1, \ldots, 1,1)$. This implies that $(W \cup x)$ is also a resolving set of $\Gamma$.

From the above cases, we conclude that the minimum cardinality of a resolving set of $\Gamma$ is $k+1$. Moreover, it is well known that every Cayley graph is a vertex transitive. Hence, the cardinality of every minimal resolving set in $\Gamma$ is $k+1$.

Theorem 6. Let $\Gamma=\operatorname{Cay}\left(\mathbb{Z}_{n}, S_{k}\right)$ be the Cayley graph on the cyclic group $\mathbb{Z}_{n}(n \geq 8)$, where $S_{1}=\{1, n-1\}, \ldots$, $S_{k}=S_{k-1} \cup\{k, n-k\}$ are the inverse closed subsets of $\mathbb{Z}_{n}-$ $\{0\}$ for any $k \in \mathbb{N}, 1 \leq k \leq[n / 2]-1$. If $n$ is an even integer and $k=(n / 2)-1$, then the cardinality of the minimum doubly resolving set of $\Gamma$ is $k+1$.

Proof. In this theorem, let $V(\Gamma)=\left\{v_{1}, v_{2}, \ldots, v_{n}\right\}$, where $v_{i}=i$ for $1 \leq i \leq n$. We know that the ordered subset $Z=$ $\{1,2,3, \ldots, k, k+1\}$ of vertices in the graph $\Gamma$ is a resolving set for $\Gamma$ of size $k+1$. Also, by Theorem 5 , the metric dimension of $\Gamma$ is $\beta(\Gamma)=k+1$. Moreover, $B(\Gamma) \leq \psi(\Gamma)$. We show that the subset $Z=\{1,2,3, \ldots, k, k+1\}$ of vertices in the graph $\Gamma$ is a doubly resolving set of $\Gamma$. It is sufficient to show that for two vertices $v_{i}$ and $v_{j}$ of $\Gamma$, there are vertices $x, y \in Z$ such that $d\left(v_{i}, x\right)-d\left(v_{i}, y\right) \neq d\left(v_{j}, x\right)-d\left(v_{j}, y\right)$. Consider two vertices $v_{i}$ and $v_{j}$ of $\Gamma$. We may assume that $i<j$. In the following cases, we show that the cardinality of the minimum doubly resolving set of $\Gamma$ is $k+1$.

Case 1. If $1 \leq i<j \leq k+1$, then $v_{i}, v_{j} \in Z$. So, $d\left(v_{i}, v_{j}\right)=1$. We can assume that $x=v_{i} \in Z$ and $y=v_{j} \in Z$. Hence, we have $d\left(v_{i}, x\right)-d\left(v_{i}\right.$, $y) \neq d\left(v_{j}, x\right)-d\left(v_{j}, y\right)$ because $d\left(v_{i}, x\right)-d\left(v_{j}, y\right)<0$ and $d\left(v_{j}, x\right)-d\left(v_{j}, y\right)>0$.

Case 2. Let $1 \leq i \leq k+1<j \leq n$. Hence, $v_{i} \in Z$ and $v_{j} \notin Z$. Moreover, we know that $d\left(v_{i}, v_{j}\right)=1$ or 2 . In the following, let $d\left(v_{i}, v_{j}\right)=1$. We may assume that $v_{i}=$ 1 and $v_{j}=n$. Hence, by taking $x=v_{i} \in Z$ and $y=$ $k+1 \in Z$, we have $0=0-0=d\left(v_{i}, x\right)-d\left(v_{i}, y\right)$ $\neq d\left(v_{j}, x\right)-d\left(v_{j}, y\right)=1-2=-1$. Thus, the vertices $x$ and $y$ of $Z$ doubly resolve $v_{i}, v_{j}$. Now, let $d\left(v_{i}, v_{j}\right)=2$. We may assume that $v_{i}=1$ and $v_{j}=k+2$. Hence, by taking $x=1 \in Z$ and $y=2 \in Z$, we have $-1=0-1=$ $d\left(v_{i}, x\right)-d\left(v_{i}, y\right) \neq d\left(v_{j}, x\right)-d\left(v_{j}, y\right)=2-1=1$. Thus, the vertices $x$ and $y$ of $Z$ doubly resolve $v_{i}, v_{j}$.

Case 3. Finally, let $1<k+1<i<j \leq n$. Hence, $v_{i} \notin Z$ and $v_{j} \notin Z$. Also, we know that $d\left(v_{i}, v_{j}\right)=1$. On the conrary, by proof of Proposition 3.2 in [27], we know that for every vertex $v_{i} \in V(\Gamma)-Z$, there is exactly one $x \in Z$ such that $d\left(v_{i}, x\right)=2$. Also, for every vertex $v_{j} \in V(\Gamma)-Z$, there is exactly one $y \in Z$ such that $d\left(v_{j}, y\right)=2$. Hence, $1=2-1=d\left(v_{i}, x\right)-d\left(v_{i}, y\right)$ $\neq d\left(v_{j}, x\right)-d\left(v_{j}, y\right)=1-2=-1$. Thus, the vertices $x$ and $y$ of $Z$ doubly resolve $v_{i}, v_{j}$.

From the above cases, we conclude that the minimum cardinality of a doubly resolving set of $\Gamma$ is $k+1$.

Theorem 7. Let $\Gamma=\operatorname{Cay}\left(\mathbb{Z}_{n}, S_{k}\right)$ be the Cayley graph on the cyclic group $\mathbb{Z}_{n}(n \geq 8)$, where $S_{1}=\{1, n-1\}, \ldots$, $S_{k}=S_{k-1} \cup\{k, n-k\}$ are the inverse closed subsets of $\mathbb{Z}_{n}-$ $\{0\}$ for any $k \in \mathbb{N}, 1 \leq k \leq[n / 2]-1$. If $n$ is an even integer and $k=(n / 2)-1$, then the strong metric dimension of $\Gamma$ is $k+1$.

Proof. In this theorem, let $V(\Gamma)=\left\{v_{1}, v_{2}, \ldots, v_{n}\right\}$, where $v_{i}=i$ for $1 \leq i \leq n$. We know that the ordered subset $N=$ $\{1,2,3, \ldots, k, k+1\}$ of vertices in the graph $\Gamma$ is a resolving set for $\Gamma$ of size $k+1$. Also, by Theorem 5 , the metric dimension of $\Gamma$ is $\beta(\Gamma)=k+1$. Moreover, $B(\Gamma) \leq \operatorname{sim}(\Gamma)$. We show that the subset $N=\{1,2,3, \ldots, k, k+1\}$ of vertices in the graph $\Gamma$ is a strong resolving set of $\Gamma$. Consider two vertices $v_{i}$ and $v_{j}$ of $\Gamma$. Assume that $i<j$. It is sufficient to prove that there exists a vertex $w \in N$ such that $v_{i}$ belongs to a shortest $v_{j}-w$ path or $v_{j}$ belongs to a shortest $v_{i}-w$ path. Let $1<k+1<i<j \leq n$. Hence, $v_{i} \notin N$ and $v_{j} \notin N$. Moreover, we have $d\left(v_{i}, v_{j}\right)=1$. On the contrary, by proof of Proposition 3.2 in [27], we know that for every vertex $v_{i} \in V(\Gamma)-N$, there is exactly one $w \in N$ such that $v_{i}^{-1} w=k+1$, indeed $d\left(v_{i}, w\right)=2$. Hence, $d\left(v_{j}, w\right)=1$. So, $d\left(v_{i}, w\right)=d\left(v_{i}, v_{j}\right)+d\left(v_{j}, w\right)$, that is, vertex $v_{j}$ belongs to a shortest $v_{i}-w$ path, and hence $w$ strongly resolves vertices $v_{i}$ and $v_{j}$. Also, for $v_{i} \in N$ or $v_{j} \in N$, vertex $v_{i}$ or vertex $v_{j}$ obviously strongly resolves pair $v_{i}, v_{j}$. Therefore, $N$ is a strong resolving set. Thus, the minimum cardinality of a strong resolving set of $\Gamma$ is $k+1$.

Conclusion 1. Let $\mathrm{CP}(n) \cong \operatorname{Cay}\left(\mathbb{D}_{2 n}, \Omega\right)$ be the Cayley graph on the dihedral group $\mathbb{D}_{2 n}$, where $\Omega$ is the inverse closed subset of $\mathbb{D}_{2 n}-\{1\}$ which is defined already. Then, the minimum cardinality of resolving set, doubly resolving set, and strong resolving set of $\mathrm{CP}(n)$ is $n$.

\section{Conclusion}

In this paper, we determined the minimum cardinality of the resolving set, doubly resolving set, and strong resolving set of the jellyfish graph JFG $(n, m)$. In particular, it has been shown that the minimum cardinality of the resolving set, doubly resolving set, and strong resolving set of the cocktail party graph $\mathrm{CP}(k+1)$ is $k+1$.

\section{Data Availability}

No data were used to support this study.

\section{Conflicts of Interest}

The authors declare that there are no conflicts of interest regarding the publication of this paper. 


\section{Acknowledgments}

The work was partially supported by the Project of Anhui Jianzhu University under grant nos. 2016QD116 and 2017dc03.

\section{References}

[1] G. Chartrand, L. Eroh, M. A. Johnson, and O. R. Oellermann, "Resolvability in graphs and the metric dimension of a graph," Discrete Applied Mathematics, vol. 105, no. 1-3, pp. 99-113, 2000.

[2] L. M. Blumenthal, Theory and Applications of Distance Geometry, Clarendon Press, Oxford, UK, 1953.

[3] F. Harary and R. A. Melter, "On the metric dimension of a graph,” Combinatoria, vol. 2, pp. 191-195, 1976.

[4] P. J. Slater, "Leaves of trees," in Proceedings of the 6th Southeastern Conference on Combinatorics, Graph theory and Computing, pp. 549-559, Boca Raton, FL, USA, 1975.

[5] R. F. Bailey and P. J. Cameron, "Base size, metric dimension and other invariants of groups and graphs," Bulletin of the London Mathematical Society, vol. 43, no. 2, pp. 209-242, 2011.

[6] P. S. Buczkowski, G. Chartrand, C. Poisson, and P. Zhang, "On $k$-dimensional graphs and their bases," Periodica Mathematica Hungarica, vol. 46, no. 1, pp. 9-15, 2003.

[7] G. G. Chappell, J. Gimbel, and C. Hartman, "Bounds on the metric and partition dimensions of a graph," Ars Combinatoria, vol. 88, pp. 349-366, 2008.

[8] M. Imran, A. Q. Baig, S. A. U. H. Bokhary, and I. Javaid, "On the metric dimension of circulant graphs," Applied Mathematics Letters, vol. 25, no. 3, pp. 320-325, 2012.

[9] Z. Jiang and N. Polyanskii, "On the metric dimension of Cartesian powers of a graph," Journal of Combinatorial Theory, Series A, vol. 165, pp. 1-14, 2019.

[10] T. Vetrík, "The metric dimension of circulant graphs," $\mathrm{Ca}-$ nadian Mathematical Bulletin, vol. 60, no. 1, pp. 206-216, 2017.

[11] Z. Beerliova, F. Eberhard, T. Erlebach et al., "Network discovery and verification," IEEE Journal on Selected Areas in Communications, vol. 24, no. 12, pp. 2168-2181, 2006.

[12] S. Khuller, B. Raghavachari, and A. Rosenfeld, "Landmarks in graphs," Discrete Applied Mathematics, vol. 70, no. 3, pp. 217-229, 1996.

[13] A. Sebö and E. Tannier, "On metric generators of graphs," Mathematics of Operations Research, vol. 29, no. 2, pp. 383393, 2004.

[14] J. Cáceres, C. Hernando, M. Mora et al., "On the metric dimension of Cartesian products of graphs," SIAM Journal on Discrete Mathematics, vol. 21, no. 2, pp. 423-441, 2007.

[15] M. Čangalović, J. Kratica, V. Kovačević-Vujčić, and M. Čangalović, "Minimal doubly resolving sets of prism graphs," Optimization, vol. 62, no. 8, pp. 1037-1043, 2013.

[16] J. Kratica, V. Kovacevic-Vujcic, M. Stojanovic, and M. Stojanović, "Minimal doubly resolving sets and the strong metric dimension of Hamming graphs," Applicable Analysis and Discrete Mathematics, vol. 6, no. 1, pp. 63-71, 2012.

[17] A. Ahmad, M. Bača, and S. Sultan, "Minimal doubly resolving sets of necklace graph," Mathematical Reports, vol. 20, no. 70, pp. 123-129, 2018.

[18] O. R. Oellermann and J. Peters-Fransen, "The strong metric dimension of graphs and digraphs," Discrete Applied Mathematics, vol. 155, no. 3, pp. 356-364, 2007.
[19] T. May and O. R. Oellermann, "The strong metric dimension of distance hereditary graphs," Journal of Combinatorial Mathematics and Combinatorial Computing, vol. 76, pp. 5973, 2011.

[20] M. Widyaningrum and T. A. Kusmayadi, "On the strong metric dimension of sun graph, windmill graph, and möbius ladder graph," Journal of Physics: Conference Series, vol. 1008, Article ID 012032, 2018.

[21] J. Kratica, M. Čangalović, and V. Kovačević-Vujčić, "Computing minimal doubly resolving sets of graphs," Computers \& Operations Research, vol. 36, no. 7, pp. 2149-2159, 2009.

[22] S. Akhter and R. Farooq, "Metric dimension of fullerene graphs," Electronic Journal of Graph Theory and Applications, vol. 7, no. 1, pp. 91-103, 2019.

[23] M. Jannesari and B. Omoomi, "The metric dimension of the lexicographic product of graphs," Discrete Mathematics, vol. 312, no. 22, pp. 3349-3356, 2012.

[24] J.-B. Liu, M. F. Nadeem, H. M. A. Siddiqui, and W. Nazir, "Computing metric dimension of certain families of toeplitz graphs," IEEE Access, vol. 7, pp. 126734-126741, 2019.

[25] S. W. Saputro, R. Simanjuntak, S. Uttunggadewa et al., "The metric dimension of the lexicographic product of graphs," Discrete Mathematics, vol. 313, no. 9, pp. 1045-1051, 2013.

[26] N. L. Biggs, Algebraic Graph Theory, Cambridge University Press, Cambridge, UK, 1993.

[27] S. M. Mirafzal and A. Zafari, "On the spectrum of a class of distance-transitive graphs," Electronic Journal of Graph Theory and Applications, vol. 5, no. 1, pp. 63-69, 2017. 